\title{
The dynamic DNA methylation landscape of the mutL homolog 1 shore is altered by MLH1-93G > A polymorphism in normal tissues and colorectal cancer
}

\author{
Andrea J. Savio ${ }^{1,2}$, Miralem Mrkonjic ${ }^{1,2}$, Mathieu Lemire ${ }^{3}$, Steven Gallinger ${ }^{1,2,3,4,5}$, Julia A. Knight 1,6
} and Bharat Bapat ${ }^{1,2,7^{*}}$

\begin{abstract}
Background: Colorectal cancers (CRCS) undergo distinct genetic and epigenetic alterations. Expression of mutL homolog 1 (MLH1), a mismatch repair gene that corrects DNA replication errors, is lost in up to 15\% of sporadic tumours due to mutation or, more commonly, due to DNA methylation of its promoter CpG island. A single nucleotide polymorphism (SNP) in the CpG island of MLH1 (MLH1-93G>A or rs1800734) is associated with CpG island hypermethylation and decreased MLH1 expression in CRC tumours. Further, in peripheral blood mononuclear cell (PBMC) DNA of both CRC cases and non-cancer controls, the variant allele of rs 1800734 is associated with hypomethylation at the MLH1 shore, a region upstream of its CpG island that is less dense in CpG sites.

Results: To determine whether this genotype-epigenotype association is present in other tissue types, including colorectal tumours, we assessed DNA methylation in matched normal colorectal tissue, tumour, and PBMC DNA from 349 population-based CRC cases recruited from the Ontario Familial Colorectal Cancer Registry. Using the semi-quantitative real-time PCR-based MethyLight assay, MLH1 shore methylation was significantly higher in tumour tissue than normal colon or PBMCs $(P<0.01)$. When shore methylation levels were stratified by SNP genotype, normal colorectal DNA and PBMC DNA were significantly hypomethylated in association with variant SNP genotype $(P<0.05)$. However, this association was lost in tumour DNA. Among distinct stages of CRC, metastatic stage IV CRC tumours incurred significant hypomethylation compared to stage I-III cases, irrespective of genotype status. Shore methylation of MLH1 was not associated with MSI status or promoter $\mathrm{CpG}$ island hypermethylation, regardless of genotype. To confirm these results, bisulfite sequencing was performed in matched tumour and normal colorectal specimens from six CRC cases, including two cases per genotype (wildtype, heterozygous, and homozygous variant). Bisulfite sequencing results corroborated the methylation patterns found by MethyLight, with significant hypomethylation in normal colorectal tissue of variant SNP allele carriers.
\end{abstract}

Conclusions: These results indicate that the normal tissue types tested (colorectum and PBMC) experience dynamic genotype-associated epigenetic alterations at the MLH1 shore, whereas tumour DNA incurs aberrant hypermethylation compared to normal DNA.

Keywords: Colorectal cancer, mutL homolog 1, Single nucleotide polymorphism, DNA methylation, Epigenetics, MethyLight, Mismatch repair, Microsatellite instability, CpG island, CpG shore

\footnotetext{
* Correspondence: bapat@lunenfeld.ca

${ }^{1}$ Lunenfeld-Tanenbaum Research Institute, Sinai Health System, 60 Murray St., Toronto, Ontario M5T 3L9, Canada

2Department of Laboratory Medicine and Pathobiology, Faculty of Medicine, University of Toronto, 27 King's College Circle, Toronto, Ontario M5S 1A1, Canada

Full list of author information is available at the end of the article
} 


\section{Background}

Colorectal cancer (CRC) develops as a result of the accumulation of genetic and epigenetic alterations. Aberrant hypermethylation of $\mathrm{CpG}$ islands along with genome-wide hypomethylation is a common signature in CRC [1,2]. While a number of genes have been shown to incur methylation in CRC, one of the best studied of these is the DNA mismatch repair (MMR) gene mutL homolog 1 (MLH1) [3-6]. Loss of MLH1 or other MMR genes leads to the accumulation of mutations, particularly at repetitive microsatellite regions leading to microsatellite instability (MSI) [6-8]. Approximately $15 \%$ of CRCs exhibit the MSI-high (MSI$\mathrm{H})$ phenotype, and the majority of these cases have deficient MMR function due to hypermethylation incurred at the $M L H 1$ promoter $\mathrm{CpG}$ island [5, 9].

Germline mutations of $M L H 1$ or other MMR genes, including mutS homolog 2 (MSH2), mutS homolog 6 (MSH6), and PMS1 homolog 2, mismatch repair system component (PMS2), lead to Lynch Syndrome accounting for approximately $2-5 \%$ of CRCs $[10,11]$. Mutations in $A P C$ cause familial adenomatous polyposis, occurring in $<1 \%$ of CRCs [12, 13]. While these and several other rarer germline gene mutations are known contributors to $\sim 10 \%$ of CRCs, twin and family studies have estimated the heritability of CRC to be up to 35\% [14]. Single nucleotide polymorphisms (SNPs) have been estimated to account for at least $7.42 \%$ of this heritability [15].

A number of genome-wide association studies (GWAS) have established susceptibility loci for CRC, including at $8 \mathrm{q} 24,11 \mathrm{q} 23$, and others [16-20]. It has previously been demonstrated that a SNP in the promoter CpG island of MLH1 (MLH1-93G>A, rs1800734) is associated with MLH1 CpG island hypermethylation, loss of protein expression, MSI, and overall increased risk of MSI-H CRC $[21,22]$. A subsequent study implicated the variant $\mathrm{A}$ allele of the SNP as contributing to increased risk of CRC overall, though another study refuted this [23, 24]. While the overall status of this SNP as a risk factor for CRC needs further clarification, what is clear is that it plays a role in MSI-H CRC and MLH1 CpG island methylation status. Interestingly, further study of SNP rs1800734 in peripheral blood mononuclear cell (PBMC) DNA using Illumina $450 \mathrm{~K}$ methylation arrays indicated a different phenomenon occurring upstream of this SNP and the CpG island in which it is located. At the MLH1 shore in PBMCs of both CRC cases and controls, we observed significant hypomethylation in association with variant SNP genotype [25].

Although the majority of DNA methylation research has focused on $\mathrm{CpG}$ islands, whole-genome methylation studies have shown that methylation changes at other non-coding regulatory regions such as CpG shores and enhancers may also be implicated in tumourigenesis
$[26,27]$. CpG shores are regions flanking some $\mathrm{CpG}$ islands that are less dense in $\mathrm{CpG}$ dinucleotides than the corresponding islands are. Differential shore methylation has been shown to discriminate between normal and tumour DNA in colorectal, prostate, and breast cancer, among other diseases [26, 28, 29].

While the mechanisms that direct DNA methylation patterns are not yet completely understood, it is guided at least in part by DNA sequence [30-33]. We have previously demonstrated DNA variant-associated CpG shore hypomethylation in PBMCs while CpG island hypermethylation was shown in CRC tumours, both of which occur in association with the same single nucleotide change [22, 25]. In this study, DNA methylation of the $M L H 1$ shore was investigated in a large cohort of 349 population-based CRC cases to determine its association with rs1800734 SNP genotype in normal colorectal tissue, colorectal tumours, and PBMCs of the same patients. These results indicate that static genetic variants can dynamically modulate epigenetic regulation at the $M L H 1$ gene region and may play a role in colorectal tumourigenesis.

\section{Methods}

Study subjects

Participants in this study were recruited through the Ontario Familial Colorectal Cancer Registry (OFCCR), which is part of the Colon Cancer Family Registry, a consortium supported by the US National Cancer Institute. Recruitment of primary CRC cases and controls was population-based and has been described previously [34]. Briefly, residents from Ontario, Canada, diagnosed with primary CRC between June 1, 1997, and June 30, 2000, between the ages of 20 and 74 were eligible for recruitment during phase I. For phase II, individuals with incident CRC under the age of 50 diagnosed in Ontario between January 2003 and December 2006 were recruited. Additional clinic-based recruitment was performed to recruit individuals diagnosed with CRC above the age of 49 with fresh frozen tumour specimens available at the biospecimen repository. Familial adenomatous polyposis cases were excluded from both phase I and II. Cases with non-white, mixed ethnic, or unknown background were excluded from the current study due to the high proportion of self-reported Caucasians. Participants provided blood, tumour, and non-neoplastic colorectal mucosa samples, henceforth referred to as normal colorectal mucosa. These blood and tissue samples were obtained with informed written consent following protocols approved by the research ethics board of Mount Sinai Hospital and the University of Toronto.

\section{Single nucleotide polymorphism genotyping}

SNP selection and genotyping has been previously described [22]. Briefly, PBMCs were isolated from blood 
samples of cases and controls by Ficoll-Paque gradient centrifugation following manufacturer's protocol (Amersham Biosciences, Baie d'Urfé, Quebec, Canada). DNA was extracted from PBMCs by phenol-chloroform or Qiagen DNA extraction kit (Qiagen Inc., Hilden, Germany). The SNP rs1800734 was genotyped using a fluorogenic $5^{\prime}$ nuclease polymerase chain reaction (PCR) assay. It was also genotyped using Affymetrix GeneChip Human Mapping $100 \mathrm{~K}$ and $500 \mathrm{~K}$ platforms through the Assessment of Risk of Colorectal Tumours in Canada project $[35,36]$. Genotypes of the five OFCCR phase II samples used for bisulfite sequencing were confirmed by Sanger sequencing at The Centre for Applied Genomics (TCAG), The Hospital for Sick Children, Toronto, Canada. DNA from samples was amplified by PCR for the region in the $M L H 1$ promoter encompassing rs1800734 using primer sequences: (forward) 5'-CGCCACATACCGCTCGTAGT A-3' and (reverse) 5'-TCCGTACCAGTTCTCAATCAT CTC-3'. Sequencing was performed at TCAG using an internal primer (forward) 5'-GTCATCCACATTCTGCG GGA-3'.

\section{Microsatellite instability analysis}

PCR was performed on tumour and matched normal colorectal tissue DNA to compare MSI patterns as described previously [35]. Briefly, paraffin-embedded colorectal tumour tissue and normal colorectal tissue from the same patients were microdissected for areas with more than $70 \%$ cellularity in tumour and normal cell populations. The MSI status was determined by using the National Cancer Institute guidelines, assessing four or more markers of ACTC, BAT-25, BAT-26, BAT-40, BAT-34C4, D10S197, D18S55, D17S250, D5S346, and MYC-L. MSI status was defined as MSI-high (MSI-H) if $\geq 30 \%$ of markers were unstable; MSI low (MSI-L) if $1-29 \%$ of markers were unstable; and microsatellite stable (MSS) if $0 \%$ of markers were unstable [36].

\section{MethyLight}

MethyLight was used to determine the DNA methylation status of the MLH1 shore in PBMCs, normal colorectal tissue, and colorectal tumours of CRC cases. Fifty nanograms of DNA was subject to bisulfite modification with the EZ DNA Methylation Gold Kit according to manufacturer's protocol (Zymo Research Corp., Orange, $\mathrm{CA})$. Primers and probe were used to amplify a region of the $M L H 1$ shore, with $A L U-C 4$ primers and probe used as control. Probes contained a $5^{\prime}$ fluorescent reporter dye and a $3^{\prime}$ quencher dye. Sequences for the MLH1 shore are as follows: (forward) 5'-ATAGTTTTGATTAA GATTAGAGGCG-3', (reverse) 5'-CGATGTTTGAATA ATTGGTTTAGG-3', and (probe) 5'-AGGCGATTTG AATTTTAGATTTTATTAACGGAA-3'. Sequences for ALU-C4 are as follows: (forward) 5'-GGTTAGGTATA
GTGGTTTATATTTGTAATTTTAGTA-3', (reverse) 5'ATTAACTAAACTAATCTTAAACTCCTAACCTCA-3' and (probe) 5'-CCTACCTTAACCTCCC-3'. Samples were analysed in duplicate in 96-well plates on an ABI 7500 RT-PCR thermocycler. Percent methylated reference (PMR) score was calculated using the following calculation: [Gene of Interest/ALU-C4] sample/[Gene of Interest $/ A L U-C 4]_{\mathrm{CpGenome}} \times 100 \%$, where CpGenome represents commercially available fully methylated CpGenome Universal Methylated DNA (Millipore, Billerica, MA). The cases selected for MethyLight profiling in this study were those from phase I OFCCR genotyped for SNP rs1800734 with available peripheral blood mononuclear cell, non-neoplastic colorectal mucosa, and tumour DNA. MLH1 CpG island methylation was determined previously using MethyLight for these cases in the same manner [22].

\section{Bisulfite sequencing}

Bisulfite sequencing was performed to analyse DNA methylation in tumour and matched normal colorectal mucosa from six CRC cases. Genomic DNA from formalin-fixed paraffin-embedded (one case from phase I OFCCR) or fresh frozen (five cases from phase II OFCCR) tissue was used. DNA was treated with EZ DNA Methylation Gold Kit for bisulfite conversion, as described previously. Primers located within the $M L H 1$ shore were designed as follows: (forward) 5'-TTTGT TTGAGAAGTGGATTGTTGTTG-3' and (reverse) $5^{\prime}-\mathrm{T}$ TTCTTCACTTAAAACTATTAAACTCC-3'. DNA was amplified by PCR for each tumour and normal colorectal sample. PCR product was purified using ChargeSwitch PCR Clean-Up Kit (Invitrogen). PCR products were cloned using the pGEM-T Easy Vector System (Promega, Madison, WI) and MAX Efficiency DH $5 \alpha$ Competent Cells (Life Technologies, Carlsbad, CA) according to manufacturer's protocol. QIAprep Spin Miniprep Kit was used according to protocol to extract plasmid DNA (Qiagen, Hilden, Germany). Each successful clone was sequenced by Sanger sequencing at TCAG and at least 15 clones were sequenced for each sample.

\section{Statistical analysis}

Sex, stage, and MSI status were compared between the cases utilized for this study and the entire OFCCR cohort by Pearson's chi-square tests. Age was compared between the two groups by Independent samples $T$ tests. MethyLight PMR values were utilized to build a multiple linear regression model to assess the relationship between methylation level at the $M L H 1$ shore and tissue type (PBMC, normal colorectal mucosa, tumour), rs1800734 genotype, age, sex, stage (TNM stage I-III compared to IV), MSI status, and MLH1 CpG island methylation status. MethyLight PMR values among 
tissue type were compared using a linear mixed model, controlling for random and fixed effects to account for different DNA sources from the same individual. PMR values between genotypes were compared using ANOVA and independent samples $T$ tests. Independent samples $T$ tests were used to compare methylation with clinicopathological variables of cases. A $6 \times 4$ contingency table and Pearson's chi-square tests were used to compare the sum of methylated CpGs between genotypes for each sample (total of 6 CpGs per clone with 15-27 clones per sample). All tests were performed using IBM SPSS Statistics 21 with two-sided $P<0.05$ defined as statistically significant.

\section{Results}

\section{Assessment of clinicopathological variables and DNA methylation}

Genotype and clinicopathological variables for the 349 CRC cases used in this study are shown in Table 1 . The cases utilized for this project constitute only a subset of the total cases recruited for the OFCCR. The 349 cases selected had been previously profiled on the Illumina Infinium HumanMethylation450 arrays, had known rs1800734 genotype status, and had available DNA from
PBMC, normal colorectal mucosa, and tumour tissue. These cases did not differ significantly from the entire cohort of cases by age, sex, stage, or MSI status.

A multiple linear regression model was built to examine the relationships between methylation levels at the MLH1 shore and rs1800734 genotype, age, sex, tumour stage, MSI status, and MLH1 CpG island methylation status (Table 2). Genotype was significantly associated with methylation $(r=-0.07, P=0.045)$. Examination of the relationships between methylation and clinicopathological variables within each tissue type was also performed. MLH1 shore methylation within PBMC DNA was significantly associated with rs1800734 genotype $(r=-0.14, P=0.02)$. Within normal colorectal tissue DNA, shore methylation was associated with SNP genotype $(r=-0.15, P=0.01)$, age $(r=0.19, P=0.001)$, and tumour stage $(r=0.13, P=0.04)$. Tumour DNA methylation was associated with tumour stage $(r=-0.14$, $P=0.02)$.

\section{The MLH1 shore is hypermethylated in tumour DNA}

MethyLight was performed to measure methylation at the MLH1 shore in DNA extracted from PBMCs,

Table 1 Distribution of clinicopathological features in primary colorectal carcinomas, including distribution among genotypes of rs1800734

\begin{tabular}{|c|c|c|c|c|}
\hline \multirow[t]{2}{*}{ Feature } & All Genotypes & GG & GA & AA \\
\hline & $N(\%)$ & $N(\%)$ & $N(\%)$ & $N(\%)$ \\
\hline Cases of primary colorectal carcinoma & 349 & $211(60.5)$ & $119(34.1)$ & $19(5.4)$ \\
\hline Mean age $( \pm S D)$ & $61.9(8.8)$ & $62.0(9.1)$ & $62.0(8.4)$ & $60.8(8.1)$ \\
\hline \multicolumn{5}{|l|}{ Sex } \\
\hline Female & $163(46.7)$ & $95(45.0)$ & $58(48.7)$ & $11(57.9)$ \\
\hline Male & $186(53.3)$ & $116(55.0)$ & $61(51.3)$ & $8(42.1)$ \\
\hline \multicolumn{5}{|l|}{ TNM stage } \\
\hline 1 & $22(6.3)$ & $15(7.1)$ & $6(5.0)$ & $1(5.3)$ \\
\hline 2 & $81(23.2)$ & $52(24.6)$ & $27(22.7)$ & $2(10.5)$ \\
\hline 3 & $208(59.6)$ & $125(59.2)$ & $70(58.8)$ & $13(68.4)$ \\
\hline 4 & $21(6.0)$ & $12(5.7)$ & $8(6.7)$ & $1(5.3)$ \\
\hline Unavailable & $17(4.9)$ & $7(3.3)$ & $8(6.7)$ & $2(10.5)$ \\
\hline \multicolumn{5}{|l|}{ MSI status } \\
\hline Stable/low & $287(82.2)$ & $181(85.8)$ & $95(79.8)$ & $11(57.9)$ \\
\hline High & $62(17.8)$ & $30(14.2)$ & $24(20.2)$ & $8(42.1)$ \\
\hline \multicolumn{5}{|l|}{ MLH1 CpG island } \\
\hline Unmethylated & $300(86.0)$ & 187 (88.6) & $98(82.3)$ & $15(78.9)$ \\
\hline Methylated & $34(9.7)$ & $17(8.1)$ & $14(11.8)$ & $3(15.8)$ \\
\hline Unavailable & $15(4.3)$ & $7(3.3)$ & $7(5.9)$ & $1(5.3)$ \\
\hline \multicolumn{5}{|l|}{ MMR germline mutation } \\
\hline No & $341(97.7)$ & 209 (99.1) & $114(95.8)$ & $18(94.7)$ \\
\hline Yes & $8(2.3)$ & $2(0.9)$ & $5(4.2)$ & $1(5.3)$ \\
\hline
\end{tabular}

Percentages may not add up to 100.0 due to rounding $S D$ standard deviation 
Table 2 Multiple linear regression model for all DNA sources as well as PBMC, normal colorectal mucosa, and CRC tumour DNA

\begin{tabular}{|c|c|c|c|c|c|c|c|c|}
\hline \multirow[b]{2}{*}{ Variable } & \multicolumn{2}{|c|}{ All DNA sources } & \multicolumn{2}{|l|}{ PBMC } & \multicolumn{2}{|c|}{ Normal colon } & \multicolumn{2}{|c|}{ Tumour } \\
\hline & $r$ & $P$ & $r$ & $P$ & $r$ & $P$ & $r$ & $P$ \\
\hline Tissue & 0.06 & 0.07 & & & & & & \\
\hline rs1800734 & -0.07 & 0.045 & -0.14 & 0.02 & -0.15 & 0.01 & 0.03 & 0.58 \\
\hline Age & 0.07 & 0.05 & -0.05 & 0.28 & 0.19 & 0.001 & 0.04 & 0.36 \\
\hline Sex & 0.0002 & 0.78 & 0.06 & 0.47 & 0.003 & 0.77 & -0.04 & 0.54 \\
\hline Stage & -0.01 & 0.79 & 0.11 & 0.07 & 0.13 & 0.04 & -0.14 & 0.02 \\
\hline MSI status & 0.03 & 0.93 & 0.05 & 0.48 & 0.04 & 0.91 & 0.01 & 0.70 \\
\hline MLH1 CpG island methylation & -0.03 & 0.49 & -0.02 & 0.60 & -0.02 & 0.57 & -0.04 & 0.39 \\
\hline
\end{tabular}

Significant results are italicized

The Pearson correlation $(r)$ and $P$ value for each variable are described

normal colorectal tissue, and colorectal tumours of 349 CRC patients. Mean methylation was compared using a linear mixed model across the three tissue types that were analysed (Fig. 1a). Mean PMR [standard deviation (SD)] in PBMCs was $29.1 \%$ (4.5), compared to $30.5 \%$ (5.8) in normal colorectal mucosa, and $33.3 \%$ (7.2) in tumour. MLH1 shore methylation was significantly higher in tumour than normal colorectal tissue $(P=0.04)$ and PBMCs $(P=0.001)$. Mean methylation did not differ significantly between normal colorectal tissue and PBMCs $(P=0.22)$.

\section{The MLH1 shore is hypomethylated in variant SNP carriers} in normal DNA

Mean methylation at the $M L H 1$ shore was compared between each genotype of rs1800734 in PBMCs, normal colorectal tissue, and colorectal tumours by ANOVA (Fig. 1b). In PBMCs, mean methylation (SD) of GG, GA, and AA cases was $30.1 \%(4.8), 28.3 \%$ (3.6), and $22.6 \%$ (3.0), respectively. This SNP-associated hypomethylation was significant $(P=0.04)$. Comparing individual genotypes, the methylation between GG and AA genotypes as well as between GA and AA genotypes in PBMC DNA was also significantly different $(P=0.003$ and 0.02 , respectively). These findings in PBMC DNA utilizing RT-PCR-based MethyLight technique confirmed our previously published Illumina array-based results indicating SNP-associated hypomethylation of the MLH1 shore region [25]. Comparing methylation among genotypes in normal colorectal tissue, mean methylation (SD) of GG, GA, and AA cases was 32.8\% (6.5), 27.3\% (4.5), and $24.0 \%$ (3.2), respectively, and these results were also significant $(P=0.005)$. Comparing individual genotypes, the shore methylation between GG and GA genotypes as well as between GG and AA genotypes was also significantly different $(P=0.003$ and 0.002 , respectively). In tumour samples stratified by genotype, mean methylation (SD) of individuals with the wildtype GG genotype was $33.3 \%$ (7.4), while in GA individuals it was $32.8 \%$ (7.0), and $37.0 \%$ (7.3) in AA individuals. Methylation did not differ significantly in tumour DNA of CRC cases regardless of SNP rs1800734 genotype, either by all three genotypes or individual genotype comparisons (e.g., GG vs. GA) $(P>0.05)$.

Tumour hypermethylation at the MLH1 shore is driven by variant SNP allele

Mean methylation was compared within each genotype of rs1800734, shown in Fig. 1c, to examine the methylation patterns across normal and tumour DNA at the MLH1 shore. Among individuals with the wildtype GG genotype of rs1800734, mean methylation (SD) was 30.1\% (4.8) in PBMCs, 32.8\% (6.5) in normal colorectal tissue, and $33.3 \%$ (7.4) in tumour tissue, which did not differ significantly $(P=0.15)$. In heterozygous individuals carrying the GA genotype, mean methylation (SD) was 28.3\% (3.6) in PBMCs, 27.3\% (4.5) in normal colorectal tissue, and $32.8 \%$ (7.0) in tumour, which differs significantly between tissues $(P=0.008)$. Lastly, in homozygous variant individuals carrying the AA genotype, mean methylation (SD) was $22.6 \%$ (3.0) in PBMCs, $24.0 \%$ (3.2) in normal colorectal tissue, and $37.1 \%$ (7.3) in tumour tissue, which also varied significantly between tissues $(P=0.01)$. Overall, significant hypermethylation in tumours compared to normal DNA is incurred only in individuals carrying one or two variant alleles of rs1800734, either GA or AA.

\section{The MLH1 shore is hypomethylated in stage IV CRC, not associated with $\mathrm{CpG}$ island methylation or MSI}

MLH1 shore methylation in tumours was tested for associations with various clinicopathological variables of the 349 CRC cases that were assessed by MethyLight. Tumour stage and $M L H 1$ shore methylation in tumour DNA was compared. The mean methylation (SD) of stage I-III tumours was $34.1 \%$ (7.3) versus $20.8 \%$ (3.6) in stage IV cases, which was highly significantly different $\left(P=6.2 \times 10^{-5}\right)$ (Fig. 2). Hypomethylation in stage IV cases was apparent for all cases, regardless of SNP genotype. In GG cases mean methylation was 33.6\% (7.4) for 

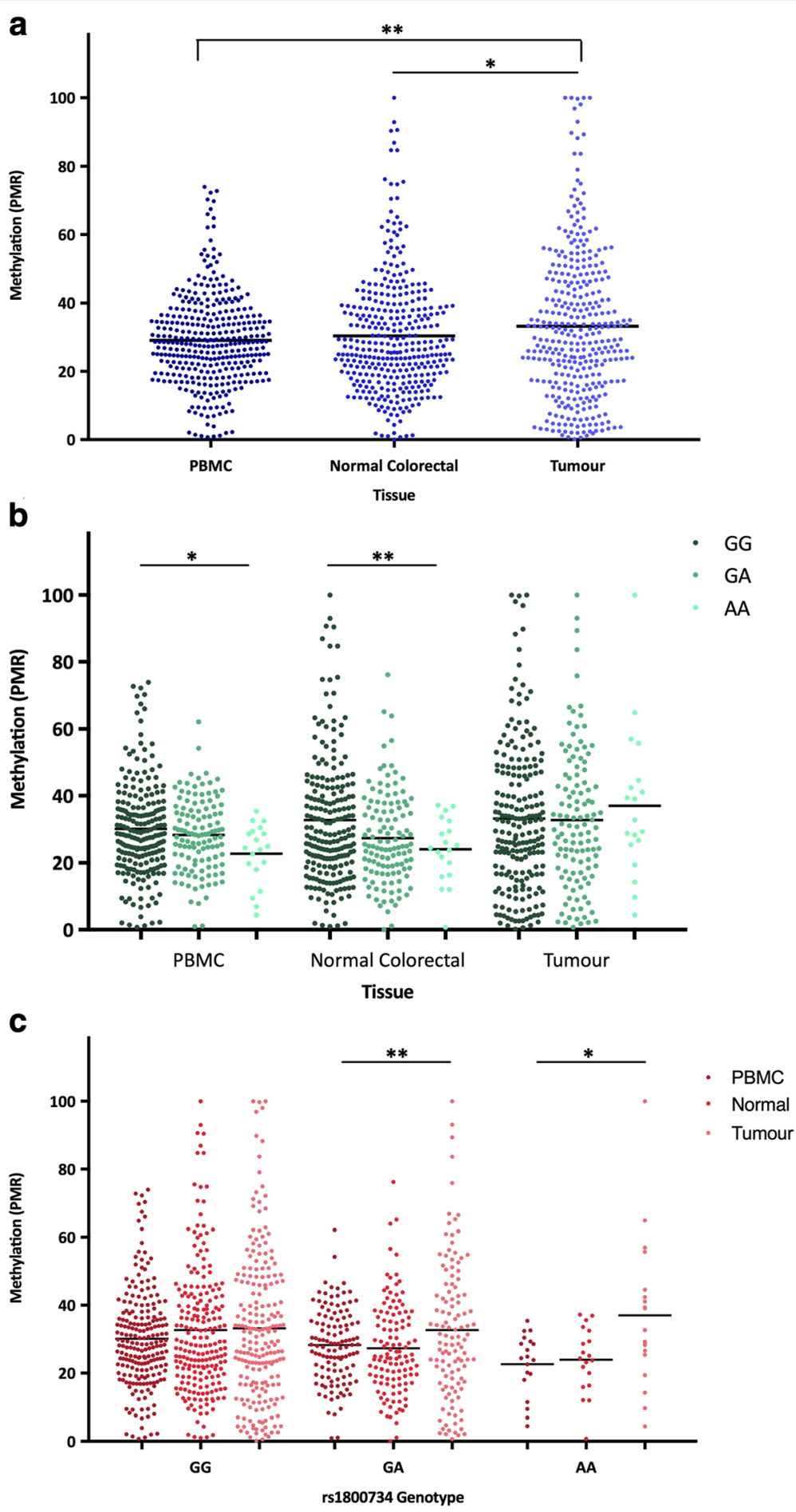

Fig. 1 Mean percent methylated reference (PMR) of the MLH1 shore. MethyLight was utilized to determine PMR in PBMC, normal colorectal tissue, and colorectal tumour DNA of 349 population-based CRC cases. All cases were genotyped for SNP rs1800734. There were 211 wildtype (GG), 119 heterozygous (GA), and 19 homozygous variant (AA) carriers. a Mean MLH1 shore methylation in each DNA source. b Mean MLH1 shore methylation in each DNA source stratified by genotype of rs1800734. c Mean MLH1 shore methylation for each genotype of rs1800734 stratified by DNA source. ${ }^{*} P<0.05$ and ${ }^{* *} P<0.01$ 


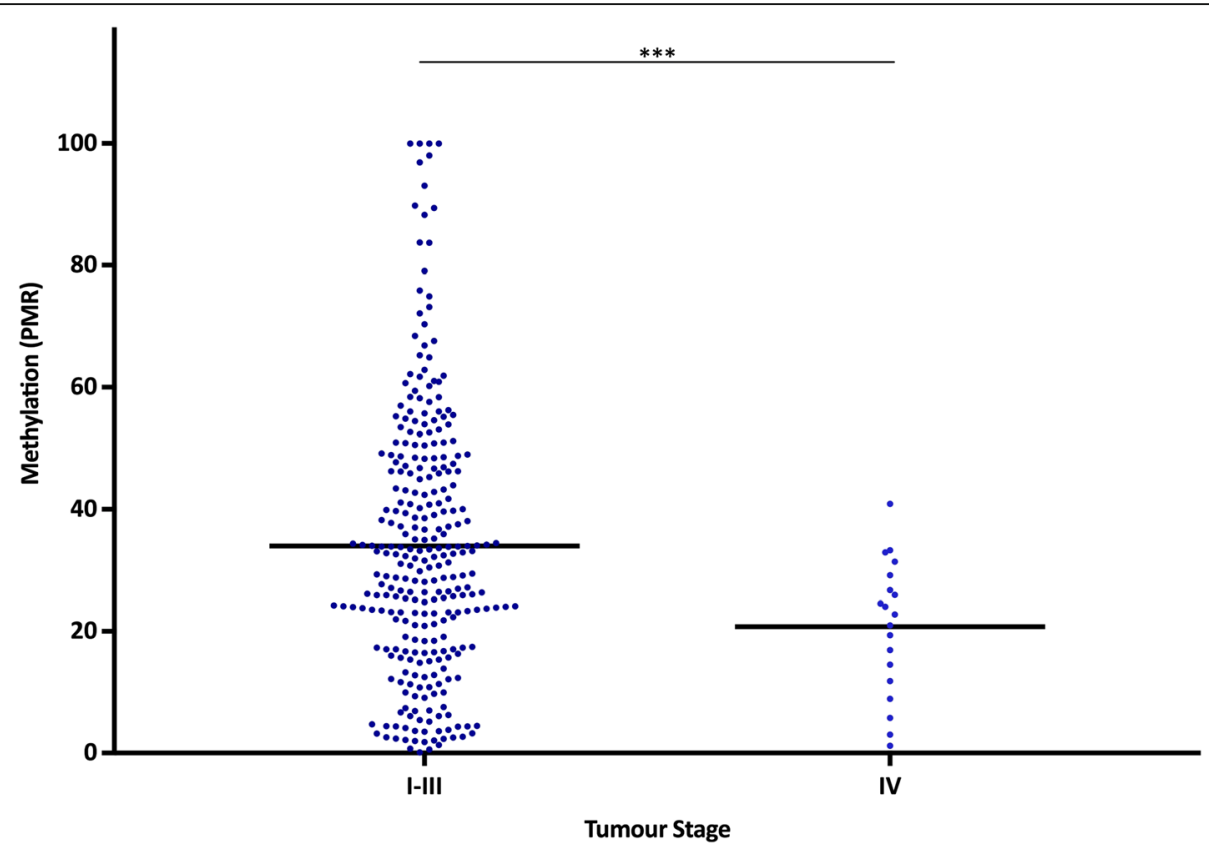

Fig. 2 Bisulfite sequencing of the MLH1 shore in normal colorectal tissue and matched colorectal tumours. a The MLH1 upstream region is indicated, including rs 1800734 coordinates. The position of each CpG interrogated by bisulfite sequencing relative to the MLH1 translation start site is designated by a circle. Each CpG is numbered 1 to 6 in a $5^{\prime}$ to $3^{\prime}$ direction. The grey circles represent CpG sites also interrogated within the MethyLight amplicon. b Methylation patterns in six normal colorectal tissue samples and matched tumours at the MLH1 shore, with rs 1800734 genotype indicated. Empty circles represent unmethylated CpGs and filled circles represent methylated CpGs. c Graphical representation of bisulfite sequencing results. For each sample at each $\mathrm{CpG}$ site, the percent of methylated CpGs was calculated. The mean percent of methylated CpGs was then calculated for each genotype and tissue source grouping: GG normal, GA normal, AA normal, GG tumour, GA tumour, and AA tumour. Pearson's chi-square test was used to compare the total number of methylated CpGs at each CpG site. Error bars represent standard deviation. **P $<0.01 ;{ }^{* * *} P<0.001$

stage I-III and $22.4 \%(4.3)$ for stage IV $(P=0.02)$. In GA cases mean methylation was $33.7 \%$ (7.1) for stage I-III and $18.4 \%(2.4)$ for stage IV $(P=0.001)$. There was only one stage IV case with the AA genotype thus no $P$ value was calculated.

Mean methylation (SD) in tumours of cases with MSI$\mathrm{H}$ phenotype was $32.9 \%$ (5.7), which did not differ from the 33.2\% (7.5) observed in MSS/MSI-L cases $(P=0.90)$. When stratified by rs1800734 SNP genotype, methylation still did not differ significantly between MSI-H and MSS/MSI-L cases (all $P>0.05$ ). We next tested whether or not methylation of the $M L H 1 \mathrm{CpG}$ island in tumour DNA was associated with $M L H 1$ shore methylation in tumour DNA of the same individuals. Cases were considered methylated at the MLH1 CpG island if PMR was greater or equal to $10 \%$, as has been previously established [22, 37]. Mean MLH1 shore methylation was $33.4 \%$ (7.4) in tumour DNA of cases that were unmethylated at the island while methylation was $30.4 \%$ (5.9) at the shore of cases that were methylated at the $\mathrm{CpG}$ island, which did not differ significantly $(P=0.41)$. Since it has previously been demonstrated that MLH1 CpG island hypermethylation is associated with rs1800734 variant genotype, we assessed whether shore methylation was associated with $\mathrm{CpG}$ island methylation stratified by genotype; however, there were no significant associations (all $P>0.05$ ). Thus, regardless of genotype, MLH1 shore methylation is not associated with $\mathrm{CpG}$ island methylation or MSI status.

MLH1 shore methylation level in PBMCs or normal colorectal tissue was not significantly associated with MSI status, tumour MLH1 CpG island hypermethylation, or tumour stage for all cases or when stratified by SNP genotype of rs1800734 (all $P>0.05$ ).

Bisulfite sequencing confirms SNP-associated hypomethylation of $M L H 1$ shore in normal colorectal DNA

Bisulfite sequencing was performed on a 232-base-pair region of the $M L H 1$ shore containing six CpGs, which we refer to as CpG 1 to 6 in a 5 to 3 direction (Fig. 3a). CpGs 2-5 were included in the MethyLight primer and probe sequences. Normal colorectal DNA and tumour DNA was analysed from six CRC cases, comprising of two samples with each genotype (GG, GA, and AA). For each sample, 15 to 27 clones were sequenced, and the methylation patterns are shown in Fig. 3b. Visual inspection of the methylation patterns 


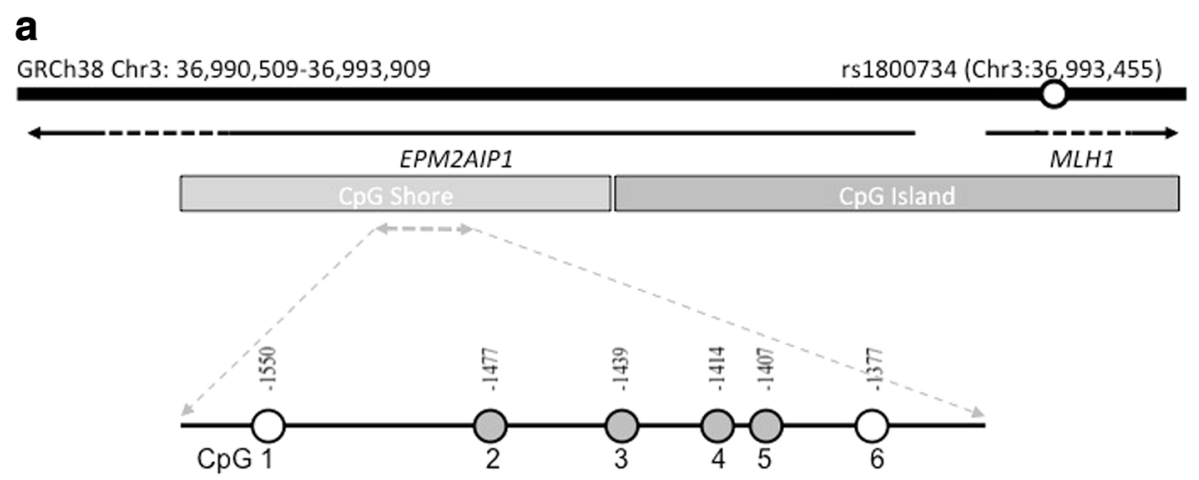

b

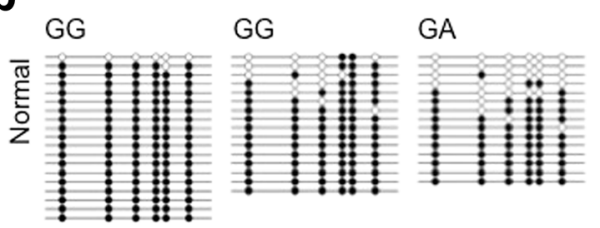

GA

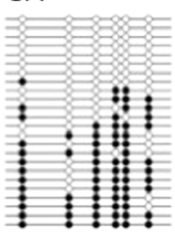

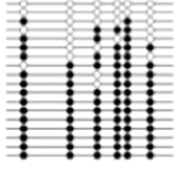

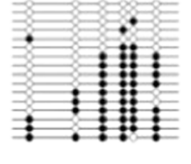

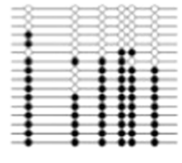

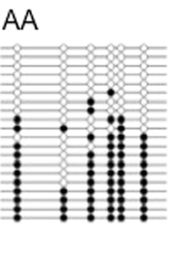

AA
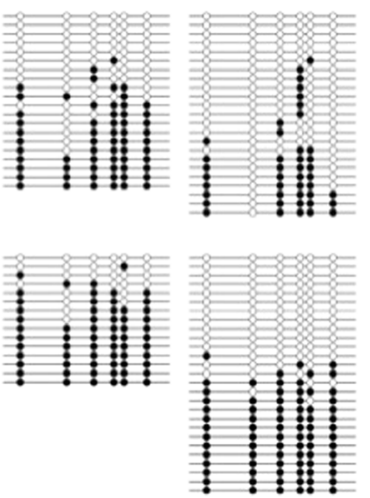

C

Normal Colorectum

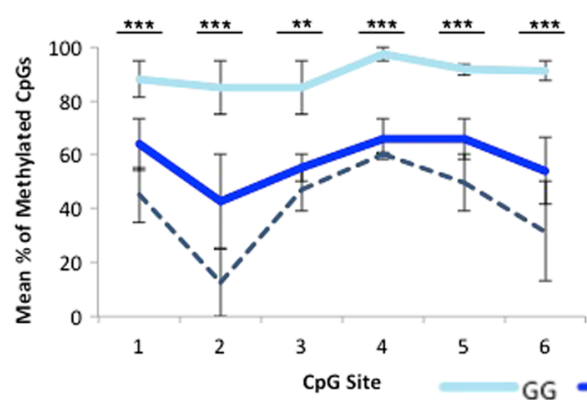

Colorectal Tumour

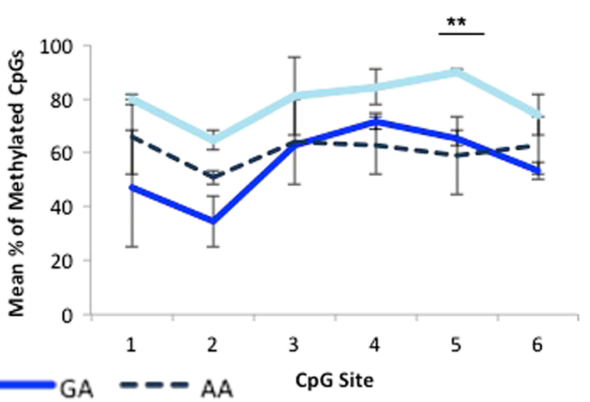

Fig. 3 Mean percent methylated reference (PMR) of the MLH1 shore among different stages in tumour DNA. MethyLight was utilized to determine PMR in colorectal tumour DNA of 349 population-based CRC cases. Mean MLH1 shore methylation among stage I-III and stage IV cases is indicated. ${ }^{* *} P<1.0 \times 10^{-4}$

shows hypomethylation in normal colorectal DNA in GA and AA cases compared to GG.

The total number of methylated CpGs at each site was compared between genotypes for normal and tumour samples, shown in Fig. 3c. All genotypes were compared against each other at CpGs 1 to 6 in normal colorectal tissue. DNA methylation as assessed by bisulfite sequencing was statistically significantly different among the three genotypes. $P$ values for each of the six $\mathrm{CpG}$ sites were CpG $1 P=2.7 \times 10^{-4}$; CpG $2 P=3.2 \times 10^{-10}$; CpG 3 $P=0.001$; CpG 4. $P=5.0 \times 10^{-4}$; CpG $5 P=3.5 \times 10^{-4}$; and CpG $6 P=3.6 \times 10^{-7}$. These results follow the same significant pattern that was observed using the MethyLight technique (Fig. 1b); normal colorectal tissue incurs hypomethylation at the $M L H 1$ shore in individuals with variant rs1800734 genotype.

The number of methylated CpGs was compared between genotypes in tumour samples. Comparing GG vs. GA vs. AA genotypes, CpG 5 was significantly differentially methylated $(P=0.002)$. Though CpG 5 showed differential methylation, all other $\mathrm{CpG}$ site comparisons between genotypes were not significantly statistically different. This corresponds with the MethyLight findings, in which tumour DNA methylation of the MLH1 
shore did not differ among genotypes of rs1800734. Methylation was also compared between normal and tumour DNA, and methylation at none of the six sites was significantly different (all $P>0.05$ ).

\section{Discussion}

Aberrant methylation changes are a hallmark of all cancers, including CRC. Though the general pattern observed in tumour DNA includes CpG island hypermethylation with genome-wide hypomethylation, the findings of the present study demonstrate that methylation may be altered in a tissue-, locus-, and genotype-specific manner. The critical mismatch repair gene $M L H 1$ incurs CpG island hypermethylation in a subset of CRC cases. We have investigated its upstream shore and determined that there is dynamic interplay between genotype and epigenotype in normal and tumour DNA of CRC patients. In normal colorectal tissue, DNA methylation is present at the CpG shore but is hypomethylated in individuals carrying one or two variant alleles of the rs1800734 SNP. This validates our previous array-based results in PBMC DNA of the same CRC cases as well as controls [25]. We also found that this SNP-associated hypomethylation pattern is lost in tumour DNA due to increases in CpG shore methylation in tumour compared to normal colorectal DNA of SNP variant carriers. These findings establish that the static genetic sequence can modulate epigenetic marks in normal tissues. These results also provide further evidence of shore methylation changes in matched tumour versus normal DNA.

This study delved into DNA methylation patterns at the $M L H 1$ shore, which builds upon previous studies of MLH1 and its promoter SNP. Variant rs1800734 was first shown to be associated with MSI-H CRCs, then with MLH1 CpG island hypermethylation [21, 22]. Subsequent studies have also shown an association between this SNP and endometrial and lung cancer risk, as well as worse outcome in oral squamous cell carcinoma [38-42]. Our results have revealed the methylation patterns among rs1800734 genotypes at the $M L H 1$ shore in PBMCs, normal colorectal tissue, and colorectal tumour tissue. We have also previously interrogated $M L H 1 \mathrm{CpG}$ island methylation in PBMC, normal colorectal, and tumour DNA [22, 25]. Figure 4 integrates methylation data from the island and shore of $M L H 1$ to demonstrate the shifting epigenetic patterns at the $M L H 1$ region. SNP genotype is associated with the opposite direction of methylation at the CpG shore and island in normal and tumour DNA.

CpG shores were first described as regions up to 2 kilobases away from CpG islands that are less dense in CpGs [26]. This original publication and subsequent studies have demonstrated that shore methylation differs between different tissue types [26, 43, 44]. However, in the two 'normal' DNA sources assessed, non-neoplastic colorectal mucosa and PBMCs, there were no significant differences in MLH1 shore methylation. Having only examined two sources of non-cancer tissues, it cannot be said for certain what methylation patterns would be seen in other normal tissues from these patients at this specific region. It has also been shown that methylation significantly differs between normal and matched tumour DNA at CpG shore regions in multiple cancer types $[26,28,29,45]$. The results in this study have indicated tumour hypermethylation at the MLH1 shore, agreeing with other reports of shore hypermethylation in cancer. Another key feature of shores is that they have been shown to have a stronger negative correlation between methylation and gene expression than $\mathrm{CpG}$ islands [26]. However, it was also found that certain subsets of genes with unmethylated islands and methylated shores, termed 'ravines', in fact had high transcriptional activity and a more transcriptionally permissive state including higher DNase sensitivity and RNA polymerase

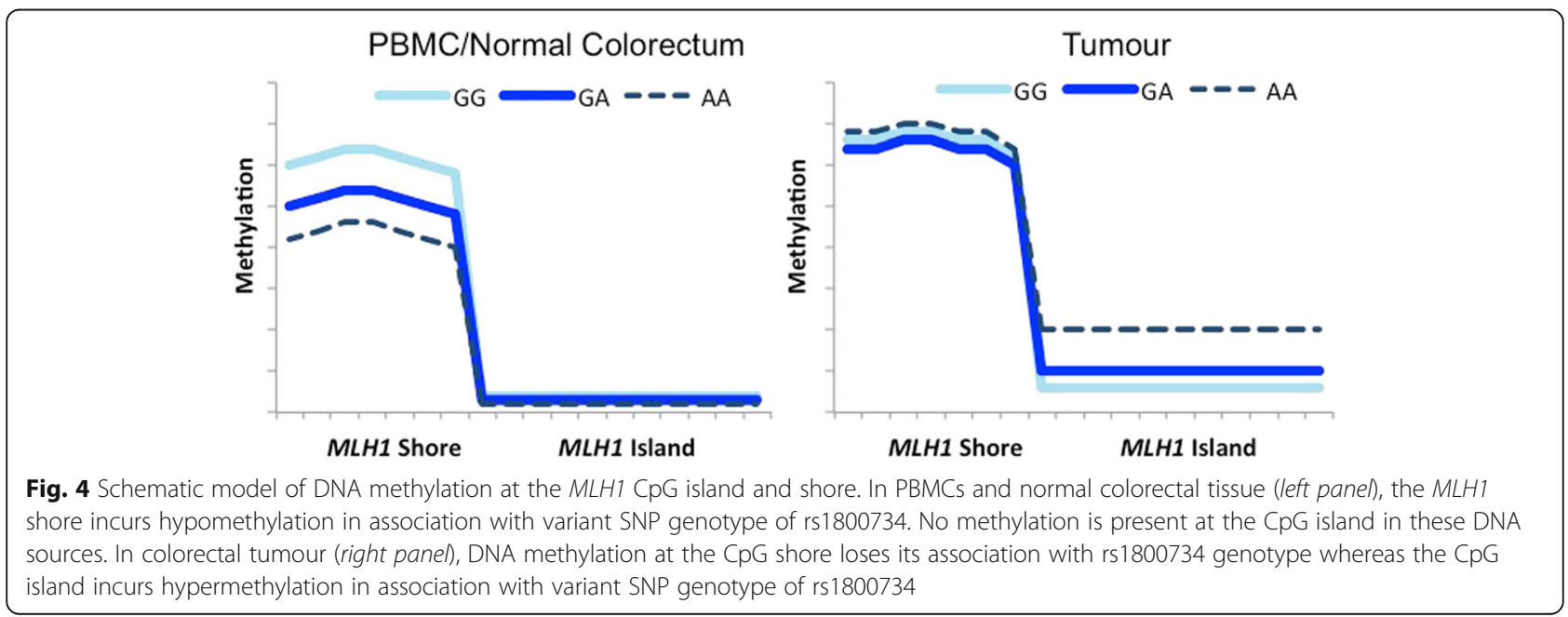


II binding [46]. In normal DNA and CRC tumours without $M L H 1$ CpG island hypermethylation, a similar pattern is seen, with methylation at the shore and no methylation at the CpG island. In fact, the $M L H 1$ promoter region has been well characterized, and the link between decreased expression and hypermethylation at specific regions in its $\mathrm{CpG}$ island have already been established [47, 48]. Thus, methylation at the MLH1 shore likely does not play a large role, if any, in $M L H 1$ expression though perhaps it functions in other ways to create a transcriptionally permissive state as in the aforementioned 'ravines' [46]. The exact mechanism or functional role for shores remains to be elucidated.

Much research has been focused toward discerning disease-associated SNPs. The majority of SNPs mapped in GWAS are located in non-coding regions of the genome, thus establishing the function of SNPs has been difficult [49-51]. Rather than altering protein function, it has been postulated that variant SNPs cause changes in gene expression levels [51, 52]. rs1800734 has not clearly emerged from CRC GWAS, though a large study has provided evidence that this SNP is a risk factor for CRC in a study of 10,409 CRC cases and 6965 controls with a significant per allele odds ratio of 1.06 [23]. However, meta-analyses have not supported these findings [23, 24]. Regardless of its influence on overall CRC incidence, substantial evidence exists for association of rs1800734 with the MSI-H subtype of CRC [21, 22]. In addition to methylation changes, we have also previously shown functional changes incurred due to the variant SNP genotype. Specifically, promoter constructs with either the $\mathrm{G}$ or $\mathrm{A}$ allele were transfected into a variety of cell lines including the CRC and normal colonic cell lines [53]. The variant A allele exhibited significantly less luciferase activity than the $\mathrm{G}$ allele in all cell lines tested. We also established, through electrophoretic mobility shift assay experiments in the CRC cell line HCT 116 and normal colonic cell line CCD-841-CoTr, the presence of a DNA-binding factor(s) with high affinity for the $\mathrm{G}$ allele but not A [51]. This work was replicated in HeLa cell nuclear extract by others [54]. Active promoters bound by transcription factors and RNA polymerase II are more resistant to incurring DNA methylation than inactive promoters $[55,56]$. Therefore, if transcription factors are unable to bind at the A allele, this likely provides the link between variant SNP genotype and increased $\mathrm{CpG}$ island methylation, but does not yet provide a mechanism for methylation changes at the shore.

These results have shown an association between a SNP and methylation at the MLH1 CpG shore in normal colorectal tissue and PBMCs, in contrast to previous studies, which demonstrated a SNP-methylation association at the $\mathrm{CpG}$ island in tumour DNA. Tumour methylation of the $\mathrm{CpG}$ island is associated with MSI-H whereas shore methylation does not show any such association. The reasons for this are unclear. Perhaps the methylation changes at the $\mathrm{CpG}$ shore and $\mathrm{CpG}$ island are two independently regulated events. Validation of these findings in other large, well-characterized CRC cohorts would confirm these SNP-associated methylation events. Additional studies of the MLH1 shore in other cancer and normal tissue types would also be of interest to determine whether this phenomenon is restricted to colorectal tissue.

We also observed stage-specific methylation changes at the MLH1 shore in tumour DNA. Specifically, hypomethylation was seen among cases with stage IV CRC, despite the fact that across all cases tumour DNA incurs hypermethylation of the $M L H 1$ shore in comparison to PBMC and normal colorectal DNA. These differences were apparent in cases with each genotype of rs1800734. These results further demonstrate the dynamic methylation patterns of the $M L H 1$ shore with respect to tumour stage, as well as SNP genotype and tissue type. Methylation studies have generally focused on hypermethylation events in cancer; however, several have investigated hypomethylation in advanced CRC, such as at LINE-1 repeats $[55,56]$. Future validation of these findings in a larger number of stage IV cases would be of value.

An advantage of this study is the availability of DNA from a large population-based cohort. This cohort is well established with DNA available from matched blood, normal colorectal tissue, and tumour, which enabled the ascertainment of a comprehensive view of methylation patterns. The methylation differences measured between genotypes were significant, but relatively subtle. For example, in normal colorectal tissue, methylation was $32.8 \%$ in GG individuals, $27.3 \%$ in GA, and $24.0 \%$ in AA. Despite this, the data still demonstrated a significant SNP association in just six samples used for the bisulfite sequencing experiments. Across multiple techniques in two types of normal DNA sources, whether a large or small sample size, the genotype-epigenotype association at the $M L H 1$ shore is significant.

A caveat to this study is that every CpG of the $M L H 1$ shore cannot be individually examined due to limitations of the techniques used. MethyLight is a real-time PCRbased method that is limited to approximately 150-basepair amplicons. This technique is only able to detect methylation if all $\mathrm{CpGs}$ in the primer and probe sequences are methylated, and does not account for variable methylation patterns. Bisulfite sequencing will account for variable methylation patterns that MethyLight cannot, and one can sequence a region up to 300 base pairs. However, this method is low throughput and not well suited for analysis of a large number of samples. Despite differences in technique, we were still able to detect significant SNP-associated hypomethylation at the 
MLH1 shore in normal DNA. Another limitation is that we only assessed methylation of the shore upstream of the MLH1 CpG island. There is also a shore downstream of MLH1 located within its coding region. We did not observe SNP-associated methylation changes in our previous methylation analysis of PBMCs in CRC cases or controls, thus we did not pursue this downstream shore in the current study [25].

\section{Conclusions}

These results demonstrate an association between the promoter polymorphism rs1800734 and DNA hypomethylation at the MLH1 shore in normal colorectal tissue, and also confirmed this in PBMCs, building upon our previous work. This association is not evident in tumour DNA from the same cases, but instead, as previously demonstrated, this polymorphism is associated with hypermethylation at the CpG island in MSI-H CRC. These results reveal that the epigenetic landscape of $M L H 1$ is dynamically regulated at least in part by the static genetic sequence. Additional characterization of epigenetic and/or transcriptional regulation at the $M L H 1 \mathrm{CpG}$ island and shore, taking into account rs1800734 genotype differences, may lead to further insight into mechanisms by which polymorphisms contribute to cancer risk.

\section{Abbreviations \\ CRC: Colorectal cancer; GWAS: Genome-wide association study; MLH1: MutL homolog 1; MMR: Mismatch repair; MSH2: MutS homolog 2; MSH6: MutS homolog 6; MSI: Microsatellite instability; MSI-H: Microsatellite instability high; MSI-L: Microsatellite instability low; MSS: Microsatellite stable; OFCCR: Ontario Familial Colorectal Cancer Registry; PBMC: Peripheral blood mononuclear cell; PCR: Polymerase chain reaction; PMR: Percent methylated reference; PMS2: PMS1 homolog 2, mismatch repair system component; SD: Standard deviation; SNP: Single nucleotide polymorphism; TCAG: The Centre for Applied Genomics}

\section{Acknowledgements}

This work was undertaken at the Department of Laboratory Medicine and Pathobiology at the University of Toronto, Toronto, Ontario, Canada, and was conducted at the Lunenfeld-Tanenbaum Research Institute, Sinai Health System, Toronto, Ontario, Canada. We sincerely thank the investigators, staff, and participants of the Colon Cancer Family Registry for their dedicated contributions leading to this work. We gratefully acknowledge The Jeremy Jass Memorial Pathology Bank for the tissue samples and pathology data used in this study.

\footnotetext{
Funding

This work was supported by grant UM1 CA167551 from the National Cancer Institute and through cooperative agreements with the following CCFR centre: Ontario Registry for Studies of Familial Colorectal Cancer (U01/U24 CA074783). This work was also supported by a Team Grant from the Canadian Institutes of Health Research (CTP-79845) awarded to BB, JAK, and SG, by the NCl under Request For Applications (CA-95-011). The content of this manuscript does not necessarily reflect the views or policies of the National Cancer Institute or any of the collaborating centres in the Colon Cancer Family Registry (CCFR) nor does mention of trade names, commercial products, or organizations imply endorsement by the US Government or CFR. The funders had no role in study design, data collection and analysis, decision to publish, or preparation of the manuscript.
}

\section{Availability of data and materials}

The datasets used and/or analysed during the current study are available from the corresponding author on reasonable request.

\section{Authors' contributions}

AJS performed methylation analysis, analysed, and interpreted data, and wrote the manuscript. MM performed SNP genotyping and MLH1 CpG island methylation analysis. ML and JAK provided statistical expertise in the analysis of results. SG provided patient samples. BB participated in the study design, data interpretation, and manuscript writing. All authors read and approved the final manuscript.

\section{Competing interests}

The authors declare that they have no competing interests.

\section{Consent for publication}

Not applicable.

\section{Ethics approval and consent to participate}

These blood and tissue samples were obtained with informed written consent following protocols approved by the research ethics board of Mount Sinai Hospital and the University of Toronto.

\section{Publisher's Note}

Springer Nature remains neutral with regard to jurisdictional claims in published maps and institutional affiliations.

\section{Author details}

${ }^{1}$ Lunenfeld-Tanenbaum Research Institute, Sinai Health System, 60 Murray St., Toronto, Ontario M5T 3L9, Canada. ${ }^{2}$ Department of Laboratory Medicine and Pathobiology, Faculty of Medicine, University of Toronto, 27 King's College Circle, Toronto, Ontario M5S 1A1, Canada. ${ }^{3}$ Ontario Institute for Cancer Research, 661 University Avenue, Toronto, Ontario M5G 0A3, Canada. ${ }^{4}$ Ontario Familial Colorectal Cancer Registry, Cancer Care Ontario, 60 Murray St., Toronto, Ontario M5T 3L9, Canada. ${ }^{5}$ Department of Surgery, University Health Network, 200 Elizabeth St., Toronto, ON M5G 2C4, Canada. ${ }^{6}$ Dalla Lana School of Public Health, University of Toronto, 155 College St., Toronto, ON M5T 3M7, Canada. ${ }^{7}$ Department of Pathology, University Health Network, 200 Elizabeth St., Toronto, ON M5G 2C4, Canada.

Received: 8 December 2016 Accepted: 2 March 2017

Published online: 09 March 2017

\section{References}

1. Yang Z, Jones A, Widschwendter M, Teschendorff AE. An integrative pancancer-wide analysis of epigenetic enzymes reveals universal patterns of epigenomic deregulation in cancer. Genome Biol. 2015;16:140.

2. Sahnane N, Magnoli F, Bernasconi B, Tibiletti MG, Romualdi C, Pedroni M, et al. Aberrant DNA methylation profiles of inherited and sporadic colorectal cancer. Clin Epigenetics. 2015;7:131.

3. Li X, Yao $X$, Wang $Y$, Hu F, Wang F, Jiang L, et al. MLH1 Promoter Methylation Frequency in Colorectal Cancer Patients and Related Clinicopathological and Molecular Features. PLoS One. 2013;8(3):e59064

4. Donehower LA, Creighton CJ, Schultz N, Shinbrot E, Gunaratne PH, Muzny $D$, et al. MLH1-silenced and non-silenced subgroups of hypermutated colorectal carcinomas have distinct mutational landscapes. J Pathol. 2014; 229(1):99-110.

5. Levine AJ, Phipps Al, Baron JA, Buchanan DD, Ahnen DJ, Cohen SA, et al. Clinicopathologic risk factor distributions for MLH1 promoter region methylation in CIMP-positive tumors. Cancer Epidemiol Biomarkers Prev. 2016;25(1):68-75.

6. Boland CR, Goel A. Microsatellite instability in colorectal cancer. Gastroenterology. 2010;138(6):2073-87. Elsevier Inc

7. Lin El, Tseng L, Gocke CD, Reil S, Le DT, Azad NS, et al. Mutational profiling of colorectal cancers with microsatellite instability. Oncotarget. 2015;6(39): 42334-44.

8. Moslein G, Tester DJ, Lindor NM, Honchel R, Cunningham JM, French AJ, et al. Microsatellite instability and mutation analysis of hMSH2 and hMLH1 in patients with sporadic, familial and hereditary colorectal cancer. Hum MolGenet. 1996;5(9):1245-52. 
9. Network CGA. Comprehensive molecular characterization of human colon and rectal cancer. Nature. 2012;487(7407):330-7.

10. Peltomäki P, Vasen $\mathrm{H}$. Mutations associated with HNPCC predisposition - update of ICG-HNPCC/INSiGHT mutation database. Dis Markers. 2004;20(4-5):269-76.

11. Aaltonen LA, Salovaara R, Kristo P, Canzian F, Hemminki A, Peltomaki $P$, et al. Incidence of hereditary nonpolyposis colorectal cancer and the feasibility of molecular screening for the disease. N Engl J Med. 1998;338(21):1481-7.

12. Tezcan G, Tunca B, Ak S, Cecener G, Egeli U, Tezcan G, et al. Molecular approach to genetic and epigenetic pathogenesis of early-onset colorectal cancer. World J Gastrointest Oncol. 2016;8(1):83-98.

13. Grady WM, Carethers JM. Genomic and epigenetic instability in colorectal cancer pathogenesis. Gastroenterology. 2008;135(4):1079-99.

14. Lichtenstein P, Holm NV, Verkasalo PK, Iliadou A, Kaprio J, Koskenvuo M, Pukkala E, Skytthe AHK. Environmental and heritable factors in the causation of cancer-analyses of cohorts of twins from Sweden, Denmark, and Finland. N Engl J Med. 2000;343(2):78-85

15. Jiao S, Peters U, Berndt S, Brenner H, Butterbach K, Caan BJ, et al. Estimating the heritability of colorectal cancer. Hum Mol Genet. 2014;23(14):3898-905.

16. Hutter CM, Slattery ML, Duggan DJ, Muehling J, Curtin K, Hsu L, et al. Characterization of the association between $8 \mathrm{q} 24$ and colon cancer: geneenvironment exploration and meta-analysis. 2010.

17. Tenesa A, Farrington SM, Prendergast JGD, Porteous ME, Walker M, Haq N, et al. Genome-wide association scan identifies a colorectal cancer susceptibility locus on $11 q 23$ and replicates risk loci at $8 q 24$ and 18q21. Nat Genet. 2009:40(5):631-7.

18. Al-Tassan NA, Whiffin N, Hosking FJ, Palles C, Farrington SM, Dobbins SE, et al. A new GWAS and meta-analysis with 1000Genomes imputation identifies novel risk variants for colorectal cancer. Sci Rep. 2015;5:10442.

19. Lemire M, Qu C, Loo LWM, Zaidi SHE, Wang H, Berndt SI, et al. A genomewide association study for colorectal cancer identifies a risk locus in $14 \mathrm{q} 23$. 1. Hum Genet. 2015;134(11-12):1249-62.

20. Biancolella M, Fortini BK, Tring S, Plummer SJ, Mendoza-Fandino GA, Hartiala J, et al. Identification and characterization of functional risk variants for colorectal cancer mapping to chromosome 11q23.1. Hum Mol Genet. 2014; 23(8):2198-209.

21. Raptis S, Mrkonjic M, Green RC, Pethe W, Monga N, Chan YM, et al. MLH1-93G>A promoter polymorphism and the risk of microsatelliteunstable colorectal cancer. J Natl Cancer Inst. 2007;99(6):463-74.

22. Mrkonjic M, Roslin NM, Greenwood CM, Raptis S, Pollett A, Laird PW, et al. Specific variants in the MLH1 gene region may drive DNA methylation, loss of protein expression, and MSI-H colorectal cancer. PLoS One. 2010;5(10):1-10

23. Whiffin N, Broderick P, Lubbe SJ, Pittman AM, Penegar S, Chandler I, et al. MLH1-93G $>$ a is a risk factor for MSI colorectal cancer. Carcinogenesis. 2011; 32(8):1157-61.

24. Chen H, Shen Z, Hu Y, Xiao Q, Bei D, Shen X, et al. Association between MutL homolog 1 polymorphisms and the risk of colorectal cancer: a metaanalysis. J Cancer Res Clin Oncol. 2015;141(12):2147-58.

25. Savio AJ, Lemire M, Mrkonjic M, Gallinger S, Zanke BW, Hudson TJ, et al. MLH1 region polymorphisms show a significant association with $\mathrm{CpG}$ island shore methylation in a large cohort of healthy individuals. PLoS One. 2012; 7(12):e51531.

26. Irizarry AR. The human colon cancer methylome shows similar hypo- and hypermethylation at conserved tissue-specific CpG island shores. Nat Genet. 2009;41(2):178-86

27. Taberlay PC, Statham AL, Kelly TK, Clark SJ, Jones PA. Reconfiguration of nucleosome-depleted regions at distal regulatory elements accompanies DNA methylation of enhancers and insulators in cancer. Genome Res. 2014; 24(9):1421-32

28. Kim JW, Kim S-T, Turner AR, Young T, Smith S, Liu W, et al. Identification of new differentially methylated genes that have potential functional consequences in prostate cancer. PLoS One. 2012;7(10):e48455.

29. Rao X, Evans J, Chae H, Kim S, Liu Y, Huang T, et al. CpG island shore methylation regulates caveolin-1 expression in breast cancer. Cancer Res. 2012;72(8 Supplement):5013-5013.

30. Lienert F, Wirbelauer C, Som I, Dean A, Mohn F, Schübeler D. Identification of genetic elements that autonomously determine DNA methylation states. Nat Genet. 2011;43(11):1091-7.

31. Cedar H, Bergman Y. Programming of DNA methylation patterns. Annu Rev Biochem. 2012;81:97-117.
32. Baubec T, Schubeler D. Genomic patterns and context specific interpretation of DNA methylation. Curr Opin Genet Dev. 2014;25(1):85-92.

33. Hernando-Herraez I, Heyn H, Fernandez-Callejo M, Vidal E, Fernandez-Bellon $\mathrm{H}$, Prado-Martinez J, et al. The interplay between DNA methylation and sequence divergence in recent human evolution. Nucleic Acids Res. 2015; 43(17):8204-14

34. Cotterchio M, McKeown-Eyssen G, Sutherland H, Buchan G, Aronson M, Easson AM, et al. Ontario familial colon cancer registry: methods and firstyear response rates. Chronic Dis Can. 2000;21(2):81-6.

35. Lindor NM, Burgart L, Leontovich O, Goldberg RM, Cunningham JM, Sargent DJ, et al. Immunohistochemistry versus microsatellite instability testing in phenotyping coloreetal tumors. J Clin Oncol. 2002;20(4):1043-8.

36. Boland C, Thibodeau S, Hamilton S, Sidranksy D, Eshleman J, Burt R, et al. A national cancer institute workshop on microsatellite instability for cancer detection and familial predisposition: development of international criteria for the determination of microsatellite instability in colorectal cancer. Cancer Res. 1998:58(22):5248-57.

37. Weisenberger DJ, Siegmund KD, Campan M, Young J, Long TI, Faasse MA, et al. CpG island methylator phenotype underlies sporadic microsatellite instability and is tightly associated with BRAF mutation in colorectal cancer. Nat Genet. 2006:38(7):787-93.

38. Poplawski T, Sobczuk A, Sarnik J, Pawlowska E, Blasiak J. Polymorphism of DNA mismatch repair genes in endometrial cancer. Exp Oncol. 2015;37(1):44-7.

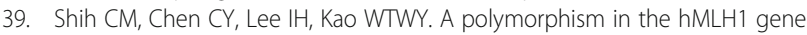
$(-93 G>A)$ associated with lung cancer susceptibility and prognosis. Int J Mol Med. 2010;25(1):165-70.

40. Lin L-H, Lin M-W, Mar K, Lin C-S, Ji D-D, Lee W-P, et al. The hMLH1 -93G>a promoter polymorphism is associated with outcomes in oral squamous cell carcinoma patients. Ann Surg Oncol. 2014;21:4270-7.

41. Yang X, Shao X, Gao L, Zhang S. Systematic DNA methylation analysis of multiple cell lines reveals common and specific patterns within and across tissues of origin. Hum Mol Genet. 2015;24(15):4374-84.

42. Ziller MJ, Gu H, Müller F, Donaghey J, Linus T. Charting a dynamic DNA methylation landscape of the human genome. Nature. 2013;500(7463):477-81.

43. Feber A, Wilson G, Zhang L, Presneau N, Idowu B, Down T, et al. Comparative methylome analysis of benign and malignant peripheral nerve sheath tumors. Genome Res. 2011;21:515-24.

44. Edgar R, Tan PPC, Portales-Casamar E, Pavlidis P. Meta-analysis of human methylomes reveals stably methylated sequences surrounding $\mathrm{CpG}$ islands associated with high gene expression. Epigenetics Chromatin. 2014;7(1):28.

45. Warnick CT, Dabbas B, Ilstrup SJ, Ford C, Strait K. Cell type-dependent regulation of hMLH1 promoter activity is influenced by the presence of multiple redundant elements. Mol Cancer Res. 2003;1:610-8.

46. Deng G, Chen A, Hong J, Chae HS, Kim YS. Methylation of CpG in a small region of the hMLH1 promoter invariably correlates with the absence of gene expression. Cancer Res. 1999;59(9):2029-33.

47. Maurano MT, Humbert R, Rynes E, Thurman RE, Wang H, Reynolds AP, et al. Systematic localization of common disease-associated variation in regulatory DNA. Science. 2012;337(6099):1190-5.

48. Butter F, Davison L, Viturawong T, Scheibe M, Vermeulen M, Todd JA, et al. Proteome-Wide Analysis of Disease-Associated SNPs That Show AlleleSpecific Transcription Factor Binding. PLoS Genet. 2012;8(9):e1002982.

49. Chen J, Tian W. Explaining the disease phenotype of intergenic SNP through predicted long range regulation. Nucleic Acids Res. 2016;44(18):8641-54.

50. Tak YG, Farnham PJ. Making sense of GWAS: using epigenomics and genome engineering to understand the functional relevance of SNPs in non-coding regions of the human genome. Epigenetics Chromatin. 2015:8(1):57.

51. Perera S, Mrkonjic M, Rawson JB, Bapat B. Functional effects of the MLH193G > A polymorphism on MLH1/EPM2AIP1 promoter activity. Oncol Rep. 2011;25(3):809-15

52. Miyakura Y, Tahara M, Lefor AT, Yasuda Y, Sugano K. Haplotype defined by the MLH1-93G/A polymorphism is associated with MLH1 promoter hypermethylation in sporadic colorectal cancers. BMC Res Notes. 2014;7:835.

53. Gebhard C, Benner C, Ehrich M, Schwarzfischer L, Schilling E, Klug M, et al. General transcription factor binding at CpG islands in normal cells correlates with resistance to de novo DNA methylation in cancer cells. Cancer Res. 2010;70(4):1398-407.

54. Takeshima H, Yamashita S, Shimazu T, Niwa T, Ushijima T. The presence of RNA polymerase II, active or stalled, predicts epigenetic fate of promoter CpG islands. Genome Res. 2009;19(11):1974-82. 
55. Kaneko M, Kotake M, Bando H, Yamada T, Takemura H, Minamoto T. Prognostic and predictive significance of long intersperced nucleotide element-1 methylation in advacned-stage colorectal cancer. BMC Cancer. 2016;16:945.

56. Hur K, Cejas P, Feliu J, Moreno-Rubio J, Burgos E, Boland CR, Goel A.

Hypomethylation of long intersperced nuclear element-1 (LINE-1) leads to activation of proto-oncogenes in human colorectal cancer metastasis. Gut. 2014;63(4):635-46.

Submit your next manuscript to BioMed Central and we will help you at every step:

- We accept pre-submission inquiries

- Our selector tool helps you to find the most relevant journal

- We provide round the clock customer support

- Convenient online submission

- Thorough peer review

- Inclusion in PubMed and all major indexing services

- Maximum visibility for your research

Submit your manuscript at www.biomedcentral.com/submit 